\title{
Staurosporine Blocks Evoked Release of FM1-43 but Not Acetylcholine from Frog Motor Nerve Terminals
}

\author{
A. W. Henkel ${ }^{\mathrm{a}}$ and W. J. Betz \\ Department of Physiology, University of Colorado Medical School, Denver, Colorado 80262
}

The protein kinase inhibitor staurosporine inhibited, and often abolished, activity-dependent destaining of frog motor nerve terminals that had been preloaded with the fluorescent dye FM1-43. Staurosporine did not, however, block synaptic transmission; staurosporine treated muscles twitched in response to nerve stimulation, and the amplitudes of evoked end plate potentials were reduced only slightly, and in some cases not at all. The blockade of FM143 destaining was not reversed by washing, although treatment with black widow spider venom caused complete destaining. Nerve terminals pretreated with staurosporine could subsequently be stained with FM1-43 (and then destained by black widow spider venom). Thus, staurosporine blocked destaining but not staining of nerve terminals. Staurosporine treatment had little effect on the ultrastructure of resting terminals, the main difference we noted being a somewhat closer packing of synaptic vesicles after exposure to staurosporine. However, staurosporine blocked completely the ultrastructural changes produced by prolonged nerve stimulation, such as depletion of synaptic vesicles, appearance of intraterminal cisternae, and the uptake of horseradish peroxidase. The effects of staurosporine were not mimicked by $\mathrm{KN}-62, \mathrm{H7}$, calmidozolium, or trifluoroperazine. These and other observations are consistent with, but do not prove the hypothesis that, after exposure to staurosporine, the exocytotic fusion pore behaved like a valve, letting FM1-43 in, but not out, as if staurosporine interfered with the postexocytotic collapse of synaptic vesicles into the surface membrane.

[Key words: neuromuscular junction, staurosporine, exocytosis, synapse, FM1-43, vesicle recycling]

FM1-43 appears to stain membranes of recycled synaptic vesicles, making it a useful tool for the study of vesicle trafficking in living nerve terminals. In frog motor nerve terminals, compact clusters of stained vesicles are imaged as fluorescent spots 1-3 $\mu \mathrm{m}$ in diameter. Spots seldom move or change shape, even dur-

Received June 7, 1995; revised Aug. 11, 1995; accepted Aug. 15, 1995

We thank Steve Fadul for his expert and enduring assistance. Drs. Wolf Almers and John Heuser provided helpful suggestions, for which we are very grateful. Ms. Dot Dill provided excellent assistance with all stages of electron microscopy. Dr. Fei Mao synthesized and kindly provided us with the FM 1-43 dimer. This work was supported by research grants from MDA and NIH (NS23466) to W.J.B. and a Human Frontiers Science Fellowship to A.W.H.

Correspondence should be addressed to Dr. W. J. Betz, Department of Physiology, Campus Box C-240, University of Colorado Medical School, Denver CO 80262

${ }^{3}$ Present address: Department of Molecular Physiology, Max-Planck Institute for Medical Research, Jahnstrasse 29, 69120 Heidelberg, Germany.

Copyright (C) 1995 Society for Neuroscience 0270-6474/95/158246-13\$05.00/0 ing tetanic nerve stimulation, which simply causes them to grow dim. This activity-dependent destaining evidently reflects exocytotic secretion of FM1-43 as dye filled vesicles move to the presynaptic membrane and undergo exocytosis, losing their dye in the process (Betz and Bewick, 1992; Betz et al., 1993; Ryan et al., 1993).

In an earlier study of the nature of synaptic vesicle clustering, we screened a number of drugs and toxins for effects on the structure of the fluorescent spots (Betz and Henkel, 1994). The most effective agent that we tested was the protein phosphatase inhibitor okadaic acid, which disrupted the fluorescent spots and caused dye (and, as seen in the electron microscope, synaptic vesicles) to disperse throughout the terminal. Dye movements were unpredictable and nonuniform, and clearly more complex than expected from simple diffusion of labeled vesicles; some regions actually became brighter after okadaic acid treatment. We interpreted these results as an unmasking by okadaic acid of a powerful and widespread mechanism for the active translocation of synaptic vesicles between vesicle clusters, the activity of which is normally suppressed (Betz and Henkel, 1994; A. W. Henkel, L. Simpson, R. M. A. P. Ridge, and W. J. Betz, unpublished observations).

As part of the okadaic acid study, we also investigated the effects of protein kinase inhibitors. One of these, staurosporine (Ruegg and Burgess, 1989), completely blocked the okadaic acid effect, as if it immobilized synaptic vesicles. Staurosporine also profoundly inhibited stimulation-induced destaining of the terminals. However, to our surprise, nerve stimulation continued to evoke muscle twitches, suggesting that synaptic transmission, unlike FM1-43 release, was not affected by staurosporine treatment. In the present study, we have confirmed and quantified those observations, and we have investigated the mechanism by which staurosporine selectively blocks destaining. Electrophysiological and optical and electron microscopic observations suggest that staurosporine may act by altering the mode of vesicle membrane internalization after exocytosis.

A preliminary of some of these findings has been published (Henkel and Betz, 1993).

\section{Materials and Methods}

Most of the methods have been described previously (Betz et al., 1992a,b). In the present study, all optical images were "top views"; that is, the nerve terminals lay on the upper surface of the most superficial muscle fibers. All experiments were performed at room temperature (about $21^{\circ} \mathrm{C}$ )

Staurosporine (Calbiochem) was dissolved in DMSO (214 $\mu 1$ of 1 $\mathrm{mm}$ staurosporine), aliquoted, and stored at $-20^{\circ} \mathrm{C}$ for up to 3 months. The standard treatment involved bathing a preparation in $2 \mu \mathrm{M}$ staurosporine in normal Ringer's for $1 \mathrm{hr}$, and then washing with normal Ringer's. The present work was performed over a period of about 30 
months. During this time, several episodes, each lasting several days, occurred during which time experimental results were inconsistent. In two such periods staurosporine blocked release by only a modest amount, significantly less than the usual $80-90 \%$. Another time, surface muscle fibers of several muscles, though of normal appearance, were dead. They did not contract and had resting potentials of $0 \mathrm{mV}$. We have not included these results in the analysis. In none of these cases were we able to identify unequivocally the source of the difficulties, since we changed several procedures simultaneously (e.g., solutions, experimental chambers, suction stimulating electrodes, stock of staurosporine). We suspect the quality or purity of the staurosporine, since we never encountered such difficulties when using freshly prepared staurosporine.

We tested several other agents, in an attempt to find other drugs that could mimic staurosporine actions. Each was applied to a prestained preparation for 45-60 min, and then the nerve was stimulated and images were acquired to measure destaining. None of the drugs tested blocked FM1-43 destaining. These included H7 (Calbiochem; $50 \mu \mathrm{M}$, which according to the supplier (Calbiochem) inhibits at this concentration several protein kinases, including PKA, PKC, and PKG, but not MLCK), KN-62 (Calbiochem; $10 \mu \mathrm{M}$, an inhibitor of calcium calmodulin kinase II), calmidozolium (RBI; $10 \mu \mathrm{M}$, a calmodulin inhibitor), and trifluoperazine (RBI; $20 \mu \mathrm{M}$, also a calmodulin inhibitor).

The procedures for preparation and use of black widow spider venom have been described (Henkel and Betz, 1995). Briefly, venom glands were homogenized in normal frog Ringer ( 1 gland $/ 0.5 \mathrm{ml}$ Ringer), vortexed for $5 \mathrm{~min}$, and centrifuged for 20 minutes at $10,000 \times \mathrm{g}$. The supernatant was collected and stored on ice. Nerve terminal destaining was assessed by incubating muscles stained with FM1-13 with freshly prepared venom ( 0.3 gland extract per $\mathrm{ml}$, dissolved in normal Ringer's) for $30-45 \mathrm{~min}$.

Standard electrophysiological techniques were used, as described previously (Betz and Bewick, 1993). Each recording obtained during prolonged stimulation of unblocked preparations (Fig. 2C,D) was examined by eye to determine if the response was an action potential (rapid repolarizing phase) or a subthreshold end plate potential (slower repolarization). When miniature end plate potentials were recorded, $1 \mu \mathrm{M}$ neostigmine was added to the bathing solution to block ACh esterase. In most experiments, to verify that the staurosporine was active, we prestained preparations with FMl-43 and, after completing electrophysiological recordings, we attempted to destain the nerve terminals by electrical stimulation. In the few cases in which terminals destained, electrophysiological results were discarded.

For dual staining, we used FM1-43 and FM4-64 (Molecular Probes). The two molecules are structurally similar. FM4-64 fluoresces at a longer wavelength owing to the presence of two additional $\mathrm{c}=\mathrm{c}$ bonds. It can be imaged best with rhodamine (red emission) optics (Heuser et al., 1993; Vida and Emr, 1995). FM1-43 is faintly visible with thodamine optics (bleedthrough), while FM4-64 shows no bleedthrough if FM143 is imaged in the green $(450-550 \mathrm{~nm}$ bandpass emission filter, instead of the usual 500-600 nm (yellow) filter). Thus, to minimize bleedthrough problems, we stained with FM4-64 and imaged first in the red, and then stained with FM1-43 and reimaged in the green (cf. Betz and Bewick, 1992). We also attempted to stain nerve terminals with other fluorophores, hoping to be able to probe the size of the fusion pore with molecules of different sizes. Dr. Fei Mao (Molecular Probes) synthesized a dinter of FM1-43, but it did not stain normal frog nerve terminals. Likewise, larger water-soluble fluorophores, such as fluorescent dextrans (Bonzelius and Zimmermann, 1990), did not stain frog terminals.

For ultrastructural studies of stimulated terminals, we stimulated muscles continuously at $10 \mathrm{~Hz}$ or $20 \mathrm{~Hz}$ for $10-15 \mathrm{~min}$, and then fixed them immediately. In all cases, we confirmed visually that muscles twitched throughout the entire stimulation period. Thus, for example, the failure of staurosporine treated preparations to take up horseradish peroxidase could not have been due to nerve block or failure to stimulate successfully. Muscles were fixed, embedded, sectioned, and examined using conventional procedures (Betz and IIenkel, 1994).

To measure uptake of horseradish peroxidase (HRP), muscles were preincubated with 5\% HRP (Sigma type VI-A) in normal frog Ringer's at room temperature, stimulated electrically via the nerve for $5 \mathrm{~min}$ at $10 \mathrm{~Hz}$, and rested for 10 additional minutes. All subsequent steps were performed at $4{ }^{\circ} \mathrm{C}$. The preparations were washed for $2 \mathrm{hr}$ in calciumfree Ringer's containing $4 \mathrm{mM} \mathrm{MgCl}_{2}$, fixed in $3 \%$ glutardaldehyde plus $2 \%$ formaldehyde (from paraformaldehyde) in $90 \mathrm{~mm}$ cacodylate buffer
(pH 7.4) for $45 \mathrm{~min}$ and washed for $2 \mathrm{hr}$ in $90 \mathrm{~mm}$ cacodylate buffer. The muscles were cut into small pieces and washed overnight in cacodylate buffer. The HRP-diaminobenzidine (DAB) reaction was initiated by preincubation of the pieces with $0.05 \% \mathrm{DAB}$ in cacodylate buffer at room temperature for $30 \mathrm{~min}$. The DAB-cacodylate buffer solution was renewed and $0.02 \% \mathrm{H}_{2} \mathrm{O}_{2}$ was added for $1 \mathrm{~min}$. The muscle pieces were then washed for $2 \mathrm{hr}$ with cacodylate buffer at $4 \mathrm{C}$, and subsequently osmium-fixed ( $2 \% \mathrm{OsO}_{4}, 20 \mathrm{mM} \mathrm{CaCl}_{2}, 50 \mathrm{~mm} \mathrm{NaCl}, 30$ mM barbital buffer, pH 7.4) in the cold. Finally they were subjected to a normal uranyl acetate block staining treatment. Control muscles were treated identically, but they were not stimulated. Staurosporine-treated muscles were incubated for $1 \mathrm{hr}$ in $2 \mu \mathrm{M}$ staurosporine prior to the start of the experiment.

To quantify numbers and spatial arrangements of synaptic vesicles, photographic negatives from the electron microscope were digitized into image files with an image scanner. The coordinates of every vesicle were then picked by hand, using a mouse-controlled cursor. In addition, the outline of the nerve terminal, and the presynaptic membrane were also digitized. Software was then written to perform the analysis of vesicle spacing and number of neighbors.

Photobleaching was performed with a laser filted on a Biorad 600 laser scanning confocal microscope (Henkel et al., unpublished observations). Images were acquired before and after small circular spots were bleached in single stained vesicle clusters.

In all graphs, results from controls are plotted with open symbols and from staurosporine treated preparations with solid symbols. Error bars show 1 SEM, unless noted otherwise.

\section{Results}

Staurosporine blocked FM1-43 destaining. Figure 1 shows results from a typical experiment in which staurosporine blocked destaining of a nerve terminal. Control terminals destained $70-$ $90 \%$ in response to the stimulation ( $30 \mathrm{~Hz}$ for $4-6 \mathrm{~min}$ ), while staurosporine treated terminals destained by only 5-20\%. In a few experiments, after confirming that electrical nerve stimulation failed to produce destaining, we exposed preparations to elevated extracellular $\left[\mathrm{K}^{+}\right]\left(60 \mathrm{mM}, \mathrm{Na}^{+}\right.$replaced by $\left.\mathrm{K}^{+}\right)$for $5-$ $10 \mathrm{~min}$. The terminals remained resistant to destaining, dimming by only about an additional $10 \%$. We tested a number of other agents (see Materials and Methods), but none of them was able to block destaining.

The block of destaining was not reversible by washing the preparation in normal Ringer's. For example, in one experiment, two muscles were stained, treated with staurosporine for one hour, washed for $1 \mathrm{hr}$, and imaged during nerve stimulation (30 $\mathrm{Hz}$ for $5 \mathrm{~min}$ ). Nerve terminals (6-8 were imaged in each muscle) destained by an average of $16 \%$. Then the preparations were washed an additional $2 \mathrm{hr}$, and the stimulation was repeated. The same terminals were then reimaged; they had destained by only an additional $12 \%$ (results not illustrated).

We wondered if staurosporine might somehow predispose terminals to phototoxic effects, which can block destaining (Betz et al., 1992a). Thus, we repeated the experiments in the dark, performing no imaging until after the nerve stimulation was completed. The results (not illustrated) were no different: staurosporine blocked destaining, whether or not preparations had been exposed to light.

In addition to blocking destaining, staurosporine also arrested movements of stained endosomes, which are often seen in Schwann cells, and can be followed most readily in time lapse movies. Staurosporine also blocked the vesicle-mobilizing effects of okadaic acid, as could be seen in both time lapse movies and in fluorescence photobleach recovery studies (Henkel et al., unpublished observations; results not illustrated here).

Staurosporine did not block evoked transmitter release. The observations described above naturally suggested that staurosporine immobilized synaptic vesicles, which might be expected 

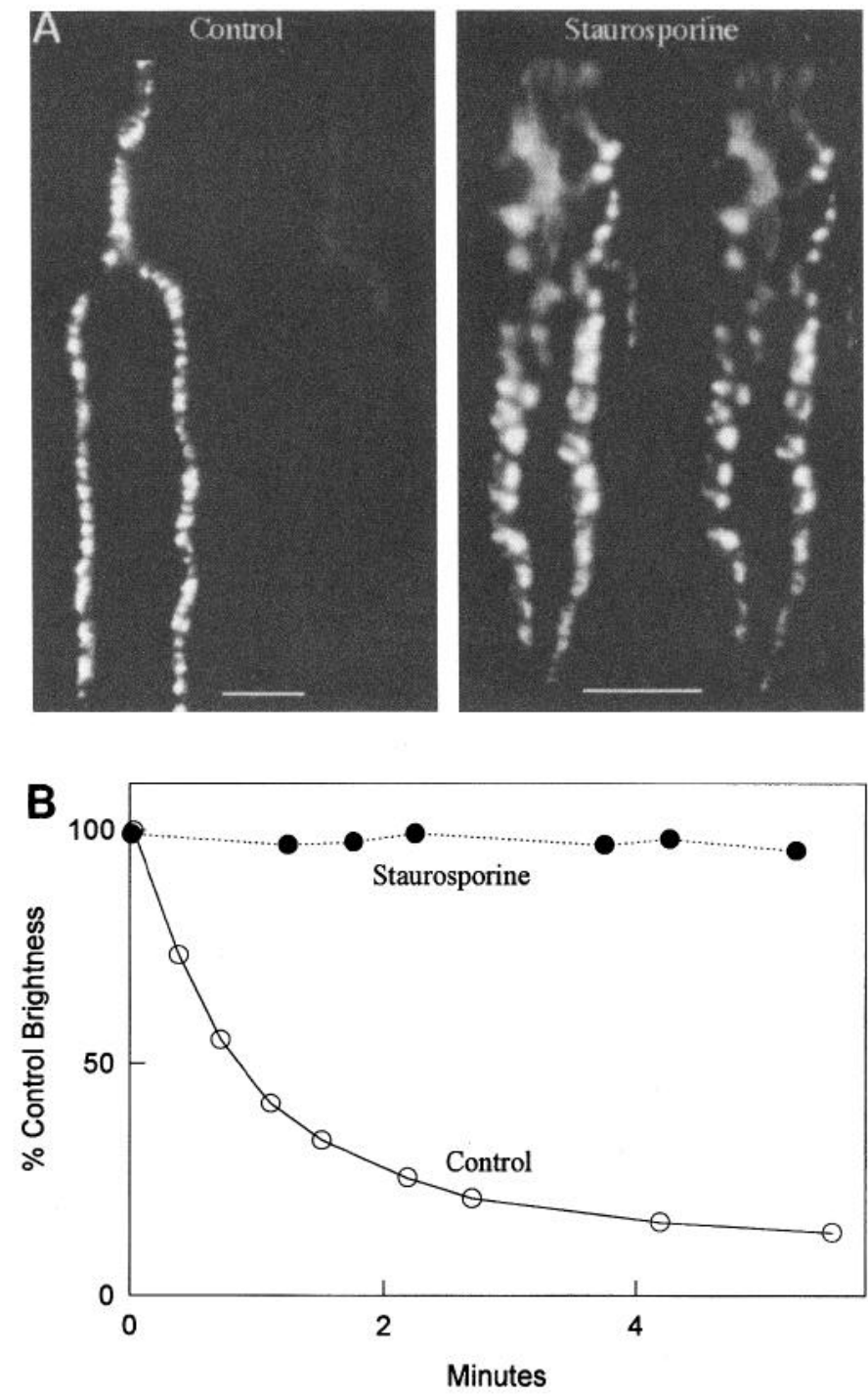

Figure 1. Staurosporine blocked activity-dependent destaining. A. Images of a control nerve terminal (left) and a staurosporine treated nerve terminal (right). In each panel, the left-hand image was acquired immediately before nerve stimulation $(30 \mathrm{~Hz}$ for $5 \mathrm{~min}$ ), and the righthand image immediately after the end of stimulation. The control terminal destained by $86 \%$, while the staurosporine treated terminal destained by only $5 \%$. Scale bar, $10 \mu \mathrm{m}$. $B$, From the same experiment as in $\mathrm{A}$, showing the time course of the changes during nerve stimulation, which began at zero minutes.

to reduce or abolish quantal release of $\mathrm{ACh}$. However, staurosporine treated muscles contracted vigorously in response to nerve stimulation (either single shocks or high frequency repetitive stimulation). It seemed unlikely that such strong contractions could have occurred if release of $\mathrm{ACh}$ had been blocked by staurosporine to the same extent as the block of FM1-43 destaining. To examine this evident discrepancy, we performed a number of electrophysiological measurements in order to quantify the staurosporine effect on transmitter release. Figure $2 A$ shows superimposed recordings of action potentials evoked by nerve stimulation in different muscle fibers impaled at random (one response per impalement). The control (left side) and staurosporine treated (right side) muscles gave very similar results. Thus, staurosporine treatment, while inhibiting FM1-43 destaining, produced virtually no block of synaptic transmission.
To quantify evoked transmitter release in more detail, action potentials and twitches were blocked by exposing preparations to curare (4-6 $\mu \mathrm{M})$, and the amplitudes of evoked end plate potentials (EPPs) were measured. Results from a typical experiment on three different muscles are shown in Figure $2 B$. First, EPPs were sampled in 15-30 cells; the three symbols near zero time show mean EPP amplitudes for each muscle. Next, two of the muscles (solid symbols) were exposed to $2 \mu \mathrm{M}$ staurosporine for about an hour. During this time evoked EPPs were recorded every minute from a single muscle fiber in each muscle; results from one of the fibers exposed to staurosporine are shown (small solid circles; similar results were obtained from fibers in the other muscles). Finally, EPPs were again sampled from 15-30 muscle fibers in each muscle; their means are shown at the right. In all three muscles, EPPs declined over time [to $46 \%$ (control) and $26 \%$ and $48 \%$ (staurosporine) of mean initial amplitude]; these declines were the result of the lack of continuous perfusion of the bathing chamber, because reperfusion with fresh Ringer's routinely caused EPP amplitudes to increase (not illustrated). In this and five other experiments, it was not possible to distinguish unequivocally control from staurosporine treated muscles by recording evoked EPPs.

We also investigated the effects of staurosporine on transmitter release during prolonged nerve stimulation (usually $10 \mathrm{~Hz}$ for $15 \mathrm{~min}$ ). Typical results from a pair of muscles are shown in Figure 2, $C$ and $D$. These experiments were performed in the absence of curare or other synaptic blocking agents to insure that all synaptic potentials were recorded. We impaled 6-8 different muscle fibers each minute during the stimulation period. Most cells, particularly at the outset of stimulation, responded with action potentials, but with continuing stimulation many responses became subthreshold. Figure $2 C$ shows that the percentage of cells giving action potentials fell progressively during the $15 \mathrm{~min}$ stimulation period, and that the responses of the control and staurosporine-treated muscles were not significantly different. We also measured amplitudes of EPPs in cells that did not respond with action potentials, as shown in Figure 2D. EPP amplitudes varied a great deal; linear regressions showed no significant difference, however, between control and staurosporine-treated preparations. Thus, as was the case for the recordings of single EPPs in curarized preparations, we could not distinguish electrophysiologically between control and staurosporine treated preparations during prolonged nerve stimulation.

Miniature end plate potential (mEPP) frequency was depressed by staurosporine. Figure $2 E$ shows results from an experiment in which we recorded $\mathrm{mEPPs}$ for $1 \mathrm{~min}$ in each of 10 muscle fibers, first in a control and then in a staurosporine treated muscle. While the average amplitudes of mEPPs were not significantly different in the two muscles, nearly three times more mEPPs occurred in the control muscle fibers. This depression of mEPP frequency by staurosporine was especially noticeable after tetanic nerve stimulation (Fig. $2 F$ ), and was the largest effect of staurosporine that we could detect with electrophysiological recordings. In two experiments, we examined the effect of elevated extracellular potassium concentration on mEPP frequency. Raising $\left[\mathrm{K}^{+}\right]_{o}$ to $30 \mathrm{mM}$ caused mEPP frequency to increase in control and staurosporine treated preparations alike (results not illustrated). Thus, as with electrical nerve stimulation of staurosporine treated preparations, high $\left[\mathrm{K}^{+}\right]_{o}$ caused little FM1-43 destaining but a large increase in ACh release.

In summary, while we did not investigate electrophysiological parameters in thorough quantitative detail, it was clear that stau- 
Fig. 2
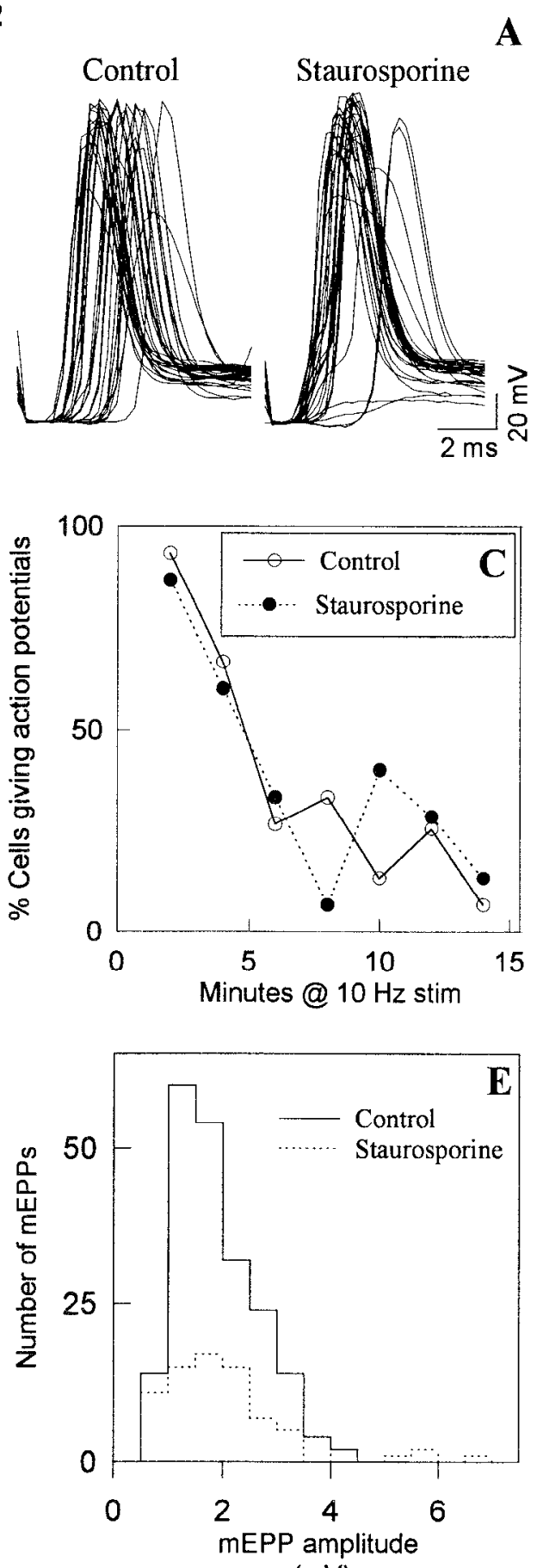
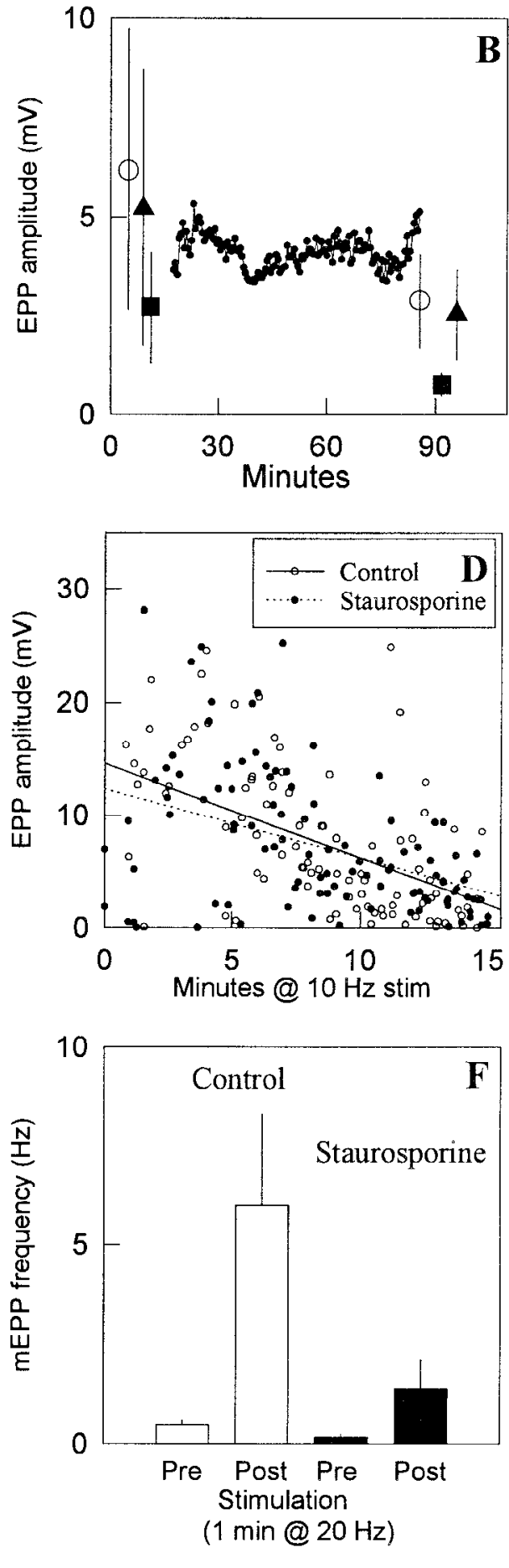

Figure 2. Staurosporine had little effect on electrophysiological measures of ACh release. A, Synaptic transmission was not blocked by staurosporine. Superimposed recordings of nerve evoked action potentials recorded from about 25 randomly impaled muscle fibers in a control muscle (left) and from a muscle pretreated with staurospotine $(r i g h t)$. For clarity, recordings are plotted as change from resting potential (resting potentials ranged from $-75 \mathrm{mV}$ (giving action potentials with the smallest amplitudes) to $-98 \mathrm{mV}$; most resting potentials fell between -90 and $-94 \mathrm{mV}$ ). $B$, EPP amplitudes were not affected by staurosporine. Curare $(5.5 \mu \mathrm{M})$ was present to block muscle action potentials and contractions. Open circles, control mean EPP amplitudes recorded from 22 muscle fibers before (left) and 24 muscle fibers after (right) a $1 \mathrm{hr}$ rest period. Solid symbols, like the control muscle, except these muscles were exposed to staurosporine for $1 \mathrm{hr}$ between the two recording sessions. Small solid circles, EPPs recorded from a single muscle fiber during exposure to staurosporine (applied after the first three EPPs had been recorded). Zero time represents, for each muscle, the time of the first impalement. $C$, Staurosporine did not alter the response to prolonged stimulation. Nerves were stimulated continuously at $10 \mathrm{~Hz}$ for $15 \mathrm{~min}$, and recordings were obtained from 6-8 muscle fibers each minute. Points show the percentage of sampled cells during successive 2 min periods that gave action potentials (the others gave subthreshold EPPs). D, As in $C$, except that the amplitudes of individual EPPs are plotted. Regression lines are nearly identical. $E$, mEPP frequency was reduced by staurosporine, but mEPP amplitudes were not affected. Amplitude histograms of all mEPPs recorded from 10 randomly impaled fibers in a control muscle (solid line) and a staurosporine treated muscle (dotted line). Each recording lasted 1 min. Control: $204 \mathrm{mEPPs}$ total, $1.40 \pm$ $1.57 \mathrm{mV}$ average amplitude. Staurosporine treated muscle: $77 \mathrm{mEPPs}$ total, $1.57 \pm 1.23 \mathrm{mV}(p>0.05)$. $F$, mEPP frequencics were lower in staurosporine treated preparations, especially after tetanic nerve stimulation. Each bar shows the mean mEPP frequency obtained by recording from $10-20$ muscle fibers for $20-60 \mathrm{sec}$ (prestimulation) or 5-20 sec (poststimulation). 

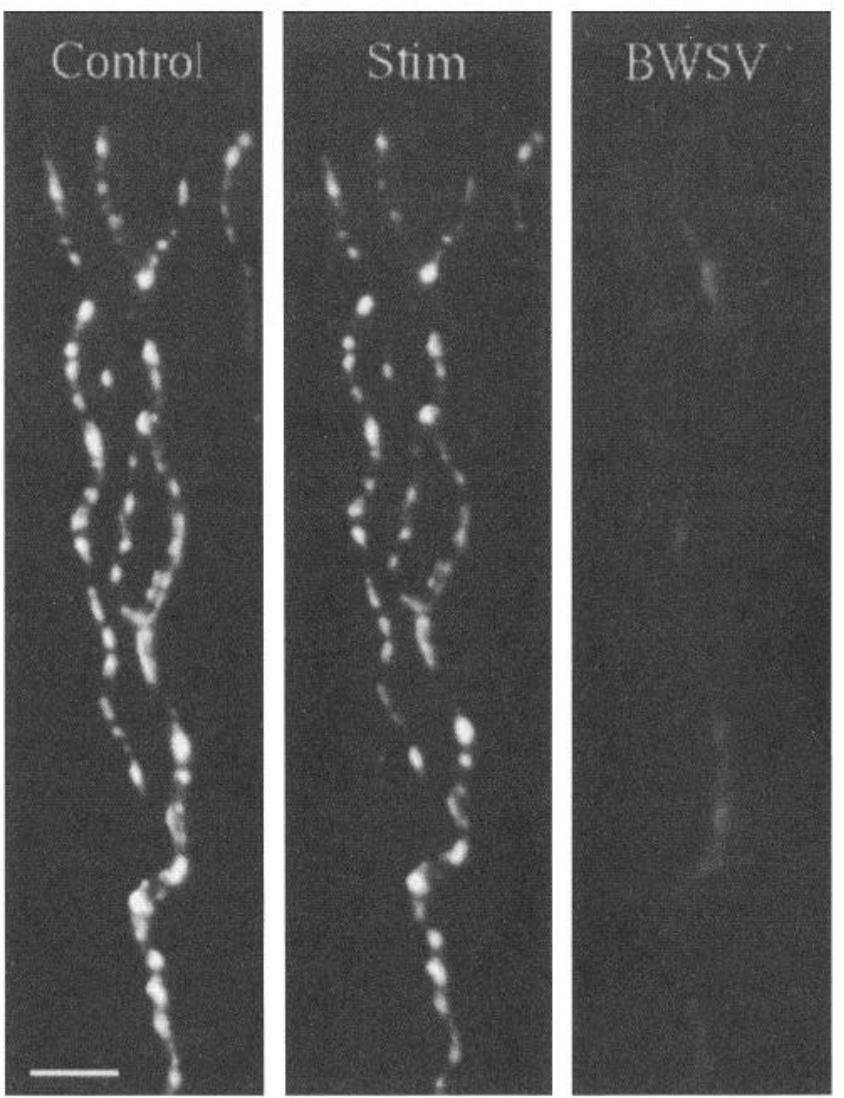

Figure 3. Black widow spider venom overcame the staurosporine-induced block of destaining. This shows a typical example of a nerve terminal that had been stained with FM1-43 and then treated with staurosporine. After the control image was acquired, the nerve was stimulated at $30 \mathrm{~Hz}$ for $4.5 \mathrm{~min}$, and another image was taken (Stim). The terminal had destained by only $19 \%$. Next, black widow spider venom was added, and $30 \mathrm{~min}$ later the last image was taken (BWSV); the terminal had destained completely. Scale bar, $5 \mu \mathrm{m}$.

rosporine treatment produced a very large discrepancy in estimates of evoked exocytosis as measured optically, by FM1- 43 destaining, and electrophysiologically, by post-synaptic potential amplitudes.

Staurosporine treated terminals were destained by black widow spider venom. One simple explanation for the results described above is that staurosporine caused FM1-43 molecules to become irreversibly bound to synaptic vesicle membranes, so that, even though vesicles may have undergone their usual cycle of exo- and endocytosis, the dye was incapable of partitioning out of the membrane into the extracellular fluid. To test this possibility, we applied black widow spider venom, a potent exocytotic agent to stained, staurosporine treated preparations (Fig. 3). Beginning about $10 \mathrm{~min}$ after black widow spider venom application, spontaneous muscle twitches could be observed, terminals started to destain, and 20-40 min later were completely destained. These effects were indistinguishable from controls. Thus, staurosporine did not appear to alter the affinity of FM143 for synaptic vesicle membranes, or, if it did, black widow spider venom was able to reverse the effect.

Preparations pretreated with staurosporine could still take up FM1-43. To see if staurosporine could block staining as well as destaining, we reversed the order of treatment, first exposing preparations to staurosporine, and then attempting to stain them with FM1-43 (Fig. 4). We found that the staining pattern in staurosporine pretreated preparations was indistinguishable from normal muscles, although the intensity of staining was usually reduced by $30-50 \%$. The terminals, once stained, were resistant to destaining by nerve stimulation (Fig. $4 A$ ). The staining after staurosporine pretreatment also was activity-dependent; exposing a staurosporine treated preparation to FM1-43 did not cause dye uptake in the absence of activity (results not illustrated).

Was the reduced staining intensity in preparations pretreated with staurosporine due to a reduction in the capacity or the rate of dye uptake during the staining procedure (or both)? To test this, we stained preparations twice in succession, washing the preparations and acquiring images after each staining period. As illustrated in Figure $4 B$, control preparations grew brighter by only $10-20 \%$ after double staining, which is consistent with earlier observations (Betz et al., 1992b) suggesting that the normal staining procedure stains most, if not all synaptic vesicles. Staurosporine-treated preparations, on the other hand, increased in brightness by $50-60 \%$ during the second staining regimen, and in most cases the terminals became as bright as controls. Thus, staurosporine treatment appeared to reduce the rate at which nerve terminals could take up FM1-43, but not their capacity to do so.

We wondered if the uptake of FM1-43 after staurosporine treatment might have been mediated by endocytosis from a "reserve" pool of surface membrane having nothing to do with the behavior of synaptic vesicles. To test this possibility, we first subjected two staurosporine treated preparations to a mock staining procedure, stimulating the nerve at $10 \mathrm{~Hz}$ for the usual 5 min, but in the absence of FM1-43. Such stimulation would presumably cause the endocytosis of any "reserve" surface membrane. Then, after a rest period, the preparations were subjected to a normal staining procedure. Terminals stained with an intensity that was not significantly different from controls (no mock staining; results not illustrated). Thus, the uptake of FM143 after staurosporine treatment was not mediated by endocytosis from a latent reservoir of surface membrane.

Finally, we measured the effects of black widow spider venom on preparations pretreated with staurosporine and then stained with FM1-43 (Fig. 4C). Like preparations treated in the reverse order, these preparations were completely destained by black widow spider venom. This suggests that FM1-43 was in the lumenal, not the cytoplasmic leaflet of the vesicle membrane (see Discussion).

In two experiments, we performed double loading experiments using dyes of different colors. If staurosporine interfered with vesicle movements, then the normal random mixing of recycled vesicles (Betz et al., 1992b) might be replaced by signs of sequestered pools of recycled vesicles. To test this, a preparation was first treated with staurosporine, then stained with FM4-64 (which fluoresces red), then imaged in the red, then stained with FM1-43 and imaged in the green (which completely excludes FM4-64 fluorescence; see Materials and Methods). The shapes of the fluorescent spots in the paired images of terminals were not noticeably different (results not illustrated). Thus, while these observations were not quantified, we saw no signs of strict queuing or other forms of sequestration of recycled vesicles in staurosporine treated terminals.

In summary, staurosporine treatment slowed by a moderate amount the rate of FM1-43 entry into the nerve terminal. In addition, the entry appeared to be mediated by vesicles undergoing exocytosis, as in normal terminals. Thus, staurosporine 

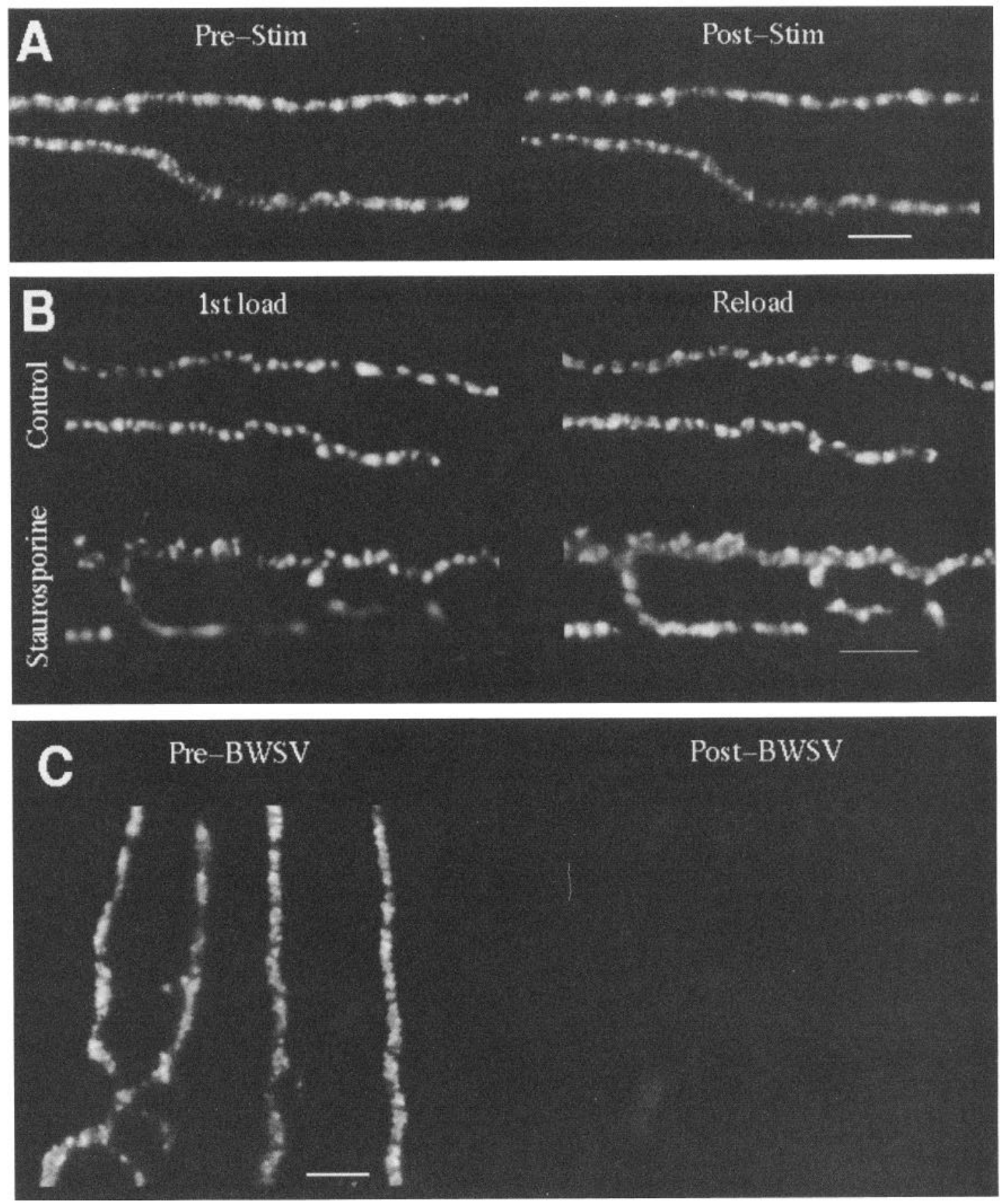

Figure 4. Terminals pretreated with staurosporine could be subsequently stained with FM1-43. A, Destaining was blocked. Typical terminal after treatment with staurosporine, followed by FM1-43 staining (Pre-Stim). After nerve stimulation ( $30 \mathrm{~Hz}$ for 5 min), this terminal had destained by $14 \%$ (Post-Stim). B, Terminals brightened significantly when stained a second time. Control (top) and staurosporine pretreated (bottom) terminals after the first loading with FM1-43 (left side) and after a second loading protocol (right side). The control terminal brightened by only $10 \%$, while the staurosporine pretreated terminal brightened by $55 \%$, after the second loading. $C$, Black widow spider venom caused destaining. Terminals were preincubated with $2 \mathrm{mM}$ staurosporine and then stained with FM1-43. Images were acquired before (Pre-BWSV) and after (Post-BWSV) exposure to black widow spider venom for $40 \mathrm{~min}$. Scale bars, $5 \mu \mathrm{m}$.

appeared to create a kind of fusion pore rectification, as if dye could get in, but not out of synaptic vesicles undergoing exocytosis.

Recovery from photobleaching did not occur in control or in staurosporine-treated terminals. We used the fluorescence photobleach recovery technique (Axelrod et al., 1976) to study dye movements in single vesicle clusters viewed from above (Fig.
5). A laser was used to bleach a segment of a fluorescent spot, and the bleached region was monitored for signs of recovery, which would reflect the movement of dye from unbleached regions into the bleached region. In separate work (Henkel et al., unpublished observations), we showed that little or no recovery from photobleaching occurs in normal terminals, either at rest or after intense nerve stimulation. Similarly, as illustrated 
Figure 5. Photobleached regions did not recover spontaneously but could be restained with FM1-43. Two examples (top and bottom) of top views of single fluorescent spots, the centers of which were bleached by a laser. Control images (left panels) were followed by images acquired immediately after bleaching (Bleach; arrowhead points to bleached region; regions bleached by $66 \%$ and $65 \%$, respectively), after nerve stimulation (Stim; $30 \mathrm{~Hz}$ for $4 \mathrm{~min}$; regions recovered by $-15 \%$ and $+19 \%$ ), and after restaining with FM1-43 (Reload; regions recovered by $86 \%$ and $85 \%$ ). The tetanic nerve stimulation caused little recovery from the photobleaching, while restaining caused the photobleached regions to disappear completely. Scale bar, $5 \mu \mathrm{m}$.
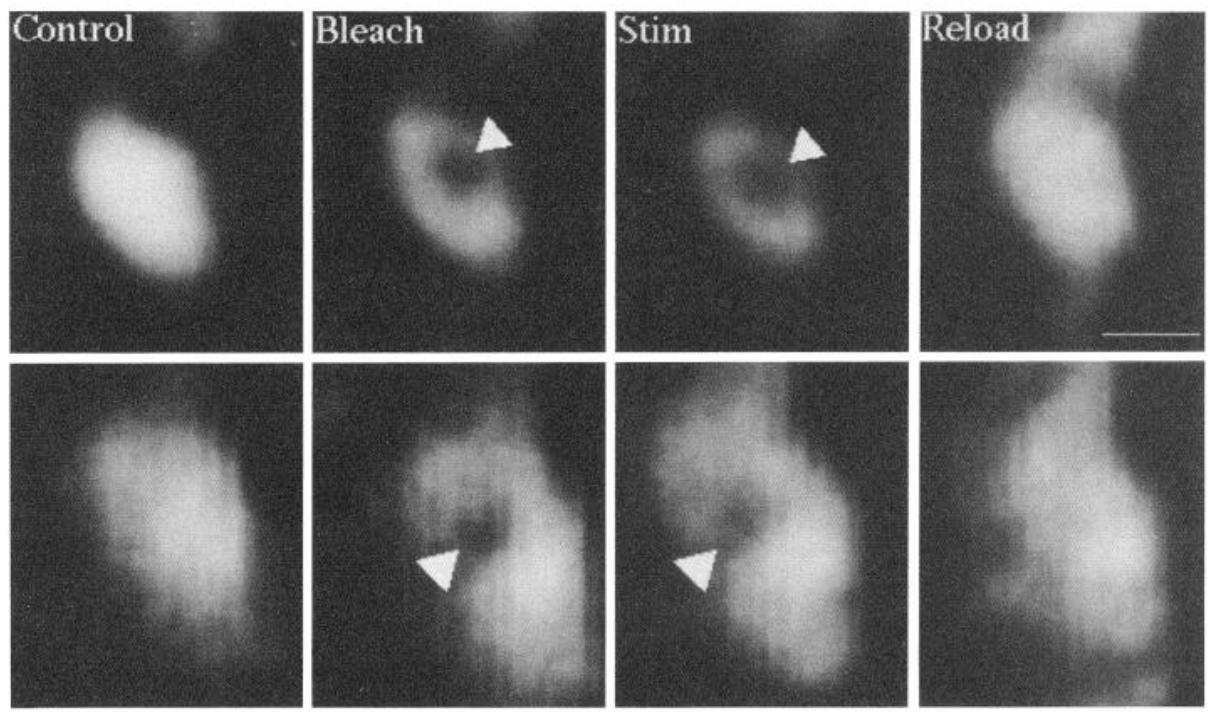

by the two examples in Figure 5, in preparations treated with staurosporine little or no recovery from photobleaching occurred, even after intense nerve stimulation. We checked for laser-induced photodamage by attempting to restain the terminals after bleaching. Results (Fig. 5) showed that the bleached regions refilled with dye. In some cases, the refilled terminals were even brighter than normal, and in others the fluorescent spots after restaining were less crisp in outline than initially. In all cases, however, the bleached regions disappeared, showing that the cytoplasmic machinery for dye uptake had not been damaged by the laser. Thus, it appears that in staurosporine treated terminals, as in normal terminals, FM1-43 dye molecules and synaptic vesicles in individual vesicle clusters are restricted in their lateral movements.

Staurosporine treatment blocked ultrastructural changes produced by nerve stimulation. We studied the effects of staurosporine on nerve terminal ultrastructure under three different conditions. First, we examined resting preparations that had been stained with FM1-43 in the usual manner, and then exposed to staurosporine. Typical examples are shown in Figure 6. General ultrastructural properties were not altered by staurosporine. Quantitative measurements showed that synaptic vesicles in staurosporine treated terminals were more tightly packed than in control terminals. In 34 cross-sectioned control nerve terminals, each synaptic vesicle had an average of $3.2 \pm 0.62$ "neighbors" (mean $\pm \mathrm{SD}$; a neighbor was defined as a synaptic vesicle closer than $60 \mathrm{~nm}$, center to center spacing); in 41 profiles of staurosporine treated nerve terminals, vesicles had $4.3 \pm 0.61$ neighbors $(p<0.01)$. The absolute numbers of synaptic vesicles per nerve terminal profile were not significantly different (control $=$ $86 \pm 51.9$ (mean $\pm 1 \mathrm{SD})$; staurosporine treated $=122 \pm 67.1$; $p>0.05)$. The tighter vesicle packing in staurosporine treated terminals, while statistically significant, was not particularly striking when examined by eye, and it was difficult to sort cor-
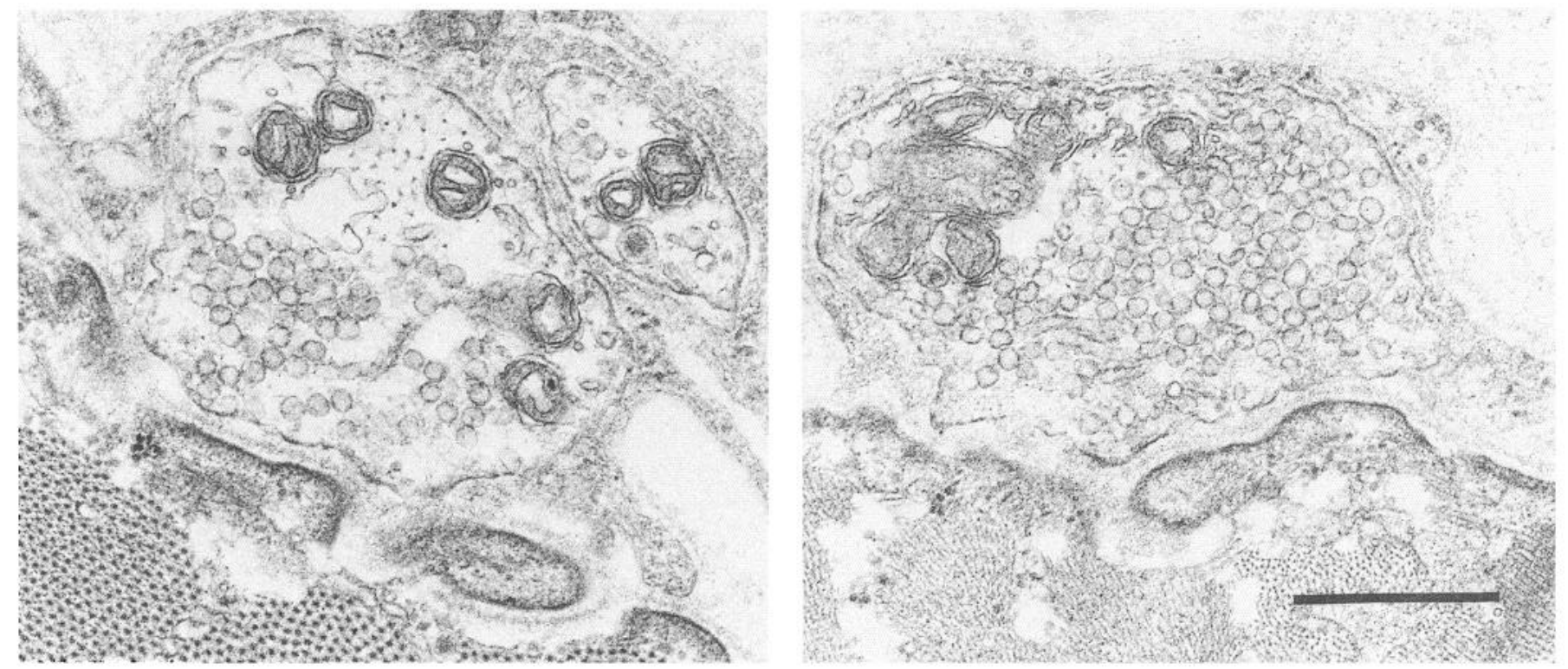

Figure 6. The ultrastructure of staurosporine treated nerve terminals was similar to controls. Typical examples of nerve terminals from a control muscle (left) and from a muscle treated with staurosporine (right). There were no discernable qualitative differences between the two types of preparations. Quantitatively, the packing of synaptic vesicles was about $30 \%$ greater in staurosporine treated preparations. Scale bar, $0.5 \mu \mathrm{m}$. 
rectly mixed electron micrographs from control and staurosporine treated preparations. In addition, we could not detect signs of tighter vesicle packing optically. That is, FM1-43 labeled vesicle clusters were not detectably brighter in staurosporine treated preparations, compared to controls (results not illustrated).

In the second ultrastructural study we examined nerve terminals that had been subjected to prolonged electrical stimulation (usually $10 \mathrm{~min}$ at $10 \mathrm{or} 20 \mathrm{~Hz}$; Fig. 7). Control preparations showed the expected changes. For example, they contained fewer synaptic vesicles and more cisternae than did unstimulated preparations. Staurosporine treatment blocked these changes; the number of synaptic vesicles was not significantly different from controls, the surface membrane did not appear distended, and increased numbers of cisternae were not apparent. Figure $7 \mathrm{~A}$ shows typical examples. We counted the number of synaptic vesicles under different conditions; results (Fig. 7B) showed that staurosporine blocked the loss of synaptic vesicles produced by prolonged stimulation.

The third ultrastructural study involved a study of horseradish peroxidase (HRP) uptake. Prolonged nerve stimulation (usually $10 \mathrm{~Hz}$ for $5 \mathrm{~min}$ ) was given in the presence of HRP. The preparation was then rested for $10 \mathrm{~min}$, washed for $2 \mathrm{hr}$ in ice-cold Ringer's without HRP, and then fixed. As shown in Figure 8, staurosporine treatment greatly reduced the uptake of HRP. In fact, we could not distinguish control, unstimulated preparations from staurosporine treated, stimulated preparations. In both cases, there was a small amount of HRP uptake, and in both cases the HRP could be found at virtually any location within the terminal. No sign of sequestration of the small amount of HRP taken up was evident in the staurosporine treated preparations.

\section{Discussion}

Staurosporine nearly abolished activity-dependent release of FM1-43, without affecting very much the quantal release of ACh. Overall, staurosporine treated terminals destained only about $15 \%$ in response to tetanic nerve stimulation; control preparations destained about 70-90\% under the same conditions. Thus, we could without fail distinguish optically a staurosporine treated preparation from a control preparation by measuring the destaining produced by nerve stimulation. Using electrophysiological techniques, however, the distinction was far more difficult. Staurosporine treatment reduced evoked release of ACh only slightly, and in some cases not at all. This result is consistent with observations on other cell types showing that staurosporine has little effect on exocytosis; these cell types include mast cells, chromaffin cells, lymphocytes, and cultured hepatocytes (Koopman and Jachson, 1990; Morgan and Burgoyne, 1990; Terbush and Holz, 1990; Fallon and Danaher, 1992; Wu et al., 1993). In at least one case, staurosporine has been reported to stimulate secretion (van der Merwe et al., 1990).

The clearest electrophysiologically detectable effect of staurosporine was a decrease in the frequency of spontaneous $m$ EPPs. This was particularly evident immediately after tetanic nerve stimulation (e.g., $20 \mathrm{~Hz}$ for $1 \mathrm{~min}$ ), when $\mathrm{mEPP}$ frequency in staurosporine treated preparations rose to only several per second, about an order of magnitude lower than in controls. Spontaneous secretion from chromaffin cells is also reduced by staurosporine (Ali and Burgoyne, 1990). Could the staurosporine-induced reduction of mEPP frequency that we observed explain the discrepant optical and electrophysiological results? This would require that, in control preparations, loss of dye is due almost entirely to the rise in MEPP frequency that occurs during tetanic stimulation. That is, activity-dependent dye loss would have to reflect exocytosis not of quanta that contribute to evoked EPPs, but to mEPPs discharged spontaneously between successive evoked EPPs. However, the contribution of such mEPPs to overall release during tetanic stimulation can be estimated conservatively to be less than $10 \%$ of the total quantal secretion of $\mathrm{ACh}$ (e.g., at $10 \mathrm{Iz}$ stimulation, assuming 30 quanta per EPP and 30 spontaneous $\mathrm{mEPPs} / \mathrm{sec}$, the total quanta/sec $=$ $10 \cdot 30+30=330$, of which $30(9 \%$ of total) are mEPPS $)$. On this basis, then, the selective blockade of spontaneous secretion (mEPPS) by staurosporine would produce, by itself, a negligible effect on nerve terminal destaining.

Alternatively, one might assume that all FM1-43 uptake and release normally occurs via asynchronous mEPPs, and that the secretory mechanism underlying evoked release is entirely refractory to staining with FM1-43. This of course would require two parallel and independent routes of quantal secretion, one for spontaneous mEPPS (which stains with FMl-43) and one for evoked quanta (which does not stain with FM1-43). Strong evidence against this is provided by the observation that destaining stops when nerve stimulation stops (Betz and Bewick, 1992), whereas the posttetanic increase in mEPP frequency persists for several minutes after the end of tetanic stimulation. Morcover, morphological evidence suggests that all, or nearly all, synaptic vesicles can be stained with horseradish peroxidase (Heuser and Reese, 1973), a result that we have recently confirmed for FM143, using the diamino benzidine photoconversion technique (Henkel, Lübke, and Betz, unpublished observations). In summary, the observed reduction in spontaneous mEPP frequency after staurosporine treatment cannot account for the profound inhibition of destaining.

How is it possible then, if molecules of FM1-43 and $\mathrm{ACh}$ coexist in the same vesicle, that one $(\mathrm{ACh})$ is released while the other (FM1-43) is not? One explanation that we tested is that staurosporine caused FM1-43 to become irreversibly linked to the synaptic vesicle membrane, so that it could not partition out of the membrane during exocytosis. Black widow spider venom, a potent trigger of synaptic vesicle exocytosis (Ceccarelli and Hurlbut, 1980), however, could overcome the block to destain ing, causing the loss of dye at a rate and to an extent that were very similar to those observed in control preparations. If black widow spider venom acted as it does in normal preparations, namely to evoke exocytosis and collapse of synaptic vesicles into the plasma membrane, then it suggests that staurosporine did not act by tethering FM1-43 molecules irreversibly to componcnts of synaptic vesicle membranes.

Another possibility is that staurosporine simply immobilized synaptic vesicles. Consistent with this, staurosporine blocks the vesicle mobilizing action of okadaic acid (Betz and Henkel, 1994) and movements of FM1-43 stained endosomes in Schwann cells. In chromaffin cells the stimulation-induced destabilization of cortical actin filaments, which may act as a barrier to the movement of granules to the plasma membrane, is also partially blocked by staurosporine, as is nicotine-induced catecholamine release (Vitale et al., 1992). However, if all vesicles are immobilized by staurosporine, then vesicular transmitter release would have to be mediated entirely by the vesicles that are "docked" at the presynaptic membrane. Docked vesicles constitute only a small percentage-less than $5 \%$ - of the total number of synaptic vesicles in a cluster. Thus, the staining by FM1-43 of a preparation pretreated with staurosporine should 
A

Figure 7. Staurosporine blocked ultrastructural changes produced by prolonged nerve stimulation. $A$, Control (top) and staurosporine treated (bottom) preparations before (left) and after (right) prolonged nerve stimulation (10 $\mathrm{Hz}$ for $10 \mathrm{~min}$ ). Before stimulation (left side) both preparations had similar appearances. After stimulation the control preparation (top right) showed the expected changes of fewer vesicles, expanded surface membrane, and more cisternae, while the staurosporine treated preparation (bottom right) resembled much more closely the control, unstimulated preparations. All preparations had been stained with FM1-43. Scale bar, $0.5 \mu \mathrm{m}$. $B$, Mean density of synaptic vesicles (number of vesicles/ area of nerve terminal) in profiles from control $(N=7)$ and staurosporine treated $(N=9)$ nerve terminals. The righthand bar is from a preparation $(N=7$ terminal profiles) that had not been stained with FM1-43.
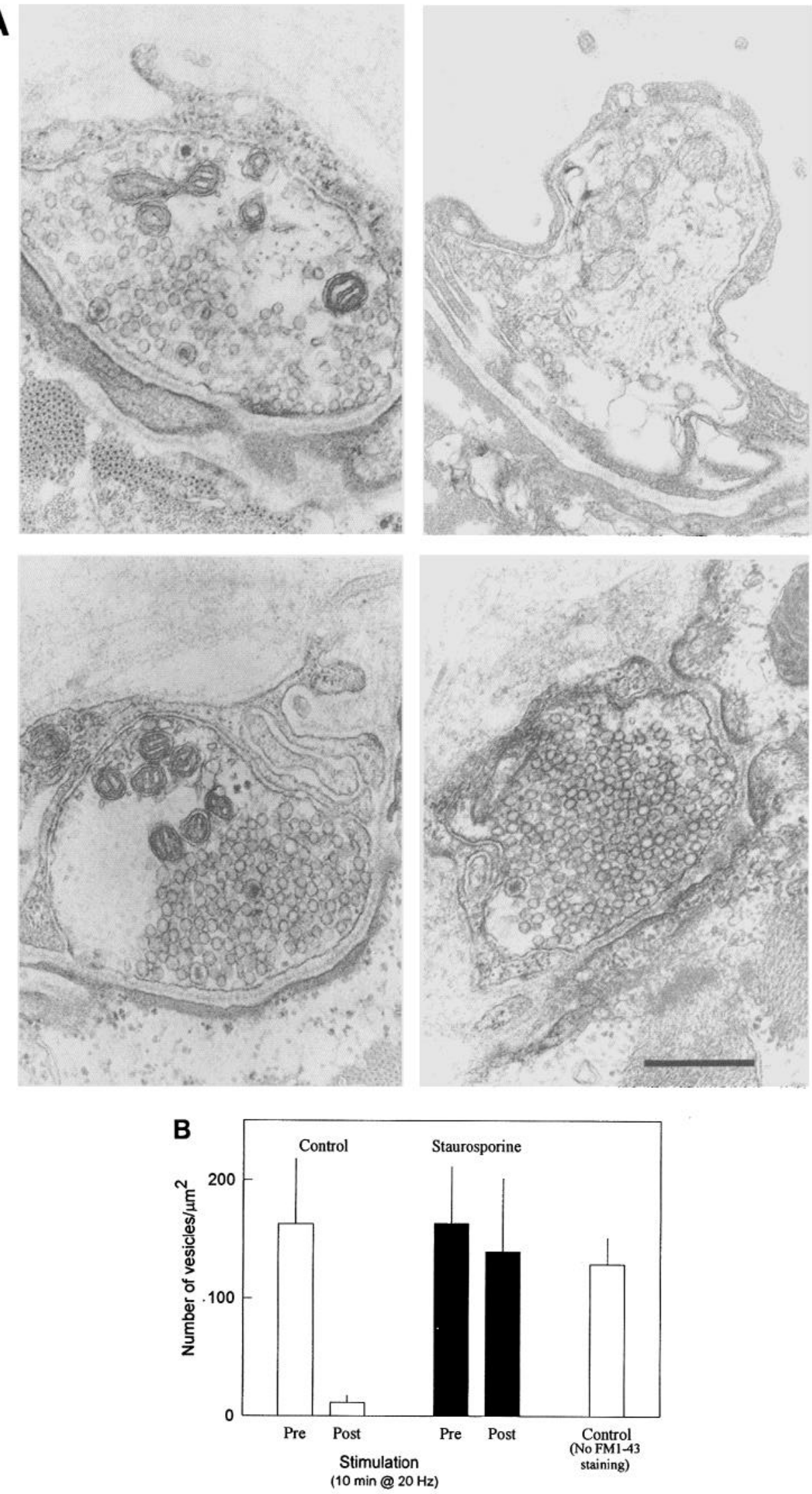

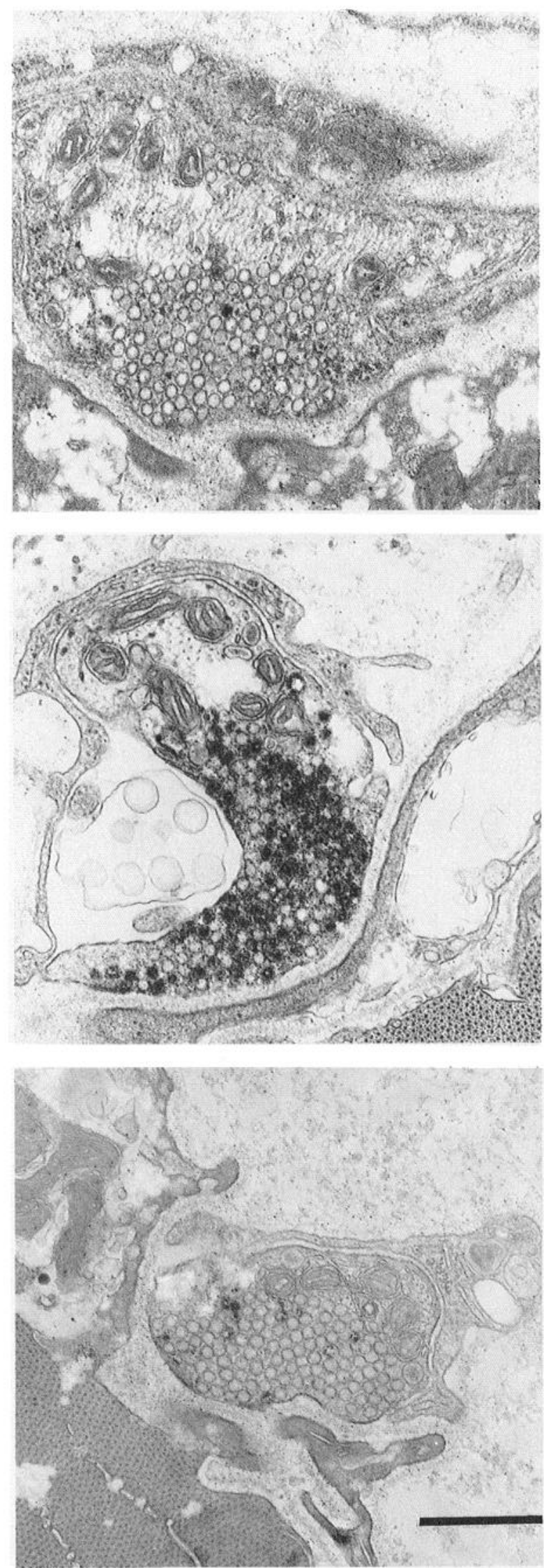

Figure 8. Staurosporine blocked the stimulation-induced uptake of horseradish peroxidase (HRP). Top panel, a control preparation was bathed in HRP for 45 minutes, without nerve stimulation. A small have been greatly reduced, compared to controls, and should have been restricted to faint stripes, about $1 \mu \mathrm{m}$ apart, marking the permanently docked vesicles at each active zone. This was not observed. The pattern of staining after staurosporine pretreatment was normal (and entirely dependent on nerve activity). The intensity of staining was reduced somewhat, but could be increased to normal levels by increasing the duration of the staining period. In summary, it appeared that staurosporine reduced the rate of, but not the capacity for, dye uptake. Thus, it seems clear that the dye had access to considerably more than $5 \%$ of the vesicle population after staurosporine treatment.

Perhaps the staining after staurosporine pretreatment reflected not the movement of vesicles, but the movement of FM1-43 molecules from one immobilized vesicle to another. One argument against such a possibility is that FM1-43 does not penetrate the nerve terminal plasma membrane and therefore should not penetrate synaptic vesicle membranes. However, significant differences in lipid composition of plasma membranes and granule membranes have been reported in chromaffin cells (Egger and Winkler, 1994), and in synaptic terminals (Benfenati et al., 1989), raising the possiblity that, once inside the terminal, FM143 might be able to move from vesicle to vesicle. However, if FM1-43 could leave a vesicle and enter the cytoplasm, it should then stain the cytoplasmic leaflet of all membranes, which would give a very different pattern of staining than that which we observed. On the other hand, our electron microscopic measurements revealed a modest but statistically significant increase in vesicle packing in staurosporine treated preparations, packing that might facilitate in some fashion the intervesicular transport of FM1-43 (cf. observations of "compound exocytosis"; Alvarez de Toledo and Fernandez, 1990; Scepek and Lindau, 1993) without widespread uptake by other intracellular organelles. To test this hypothesis directly, we used a laser to bleach a portion of a fluorescent spot, and then looked for signs of recovery from photobleaching (Axelrod et al., 1976; Jacobson et al., 1991). Little or no recovery occurred, either in normal or staurosporine treated preparations. To test for laser-induced photodamage, which might have blocked dye movements that otherwise would have occurred, we attempted to restain the bleached region with FM1-43. Restaining was successful. While this does not rule out the possibility that dye movements between vesicles was blocked by laser induced photodamage, it shows at least that the complex mechanism of dye uptake had not been damaged by the laser, and suggests that synaptic vesicles and FM1-43 molecules are not free to move laterally within a vesicle cluster.

Still another explanation for the selective block of FM1-43 destaining is that staurosporine abolished vesicular release of $\mathrm{ACh}$ altogether, and that, in response to nerve stimulation, ACh was released directly from the cytoplasm (Israel et al., 1989). In this case, FM1-43, applied after staurosporine treatment, would enter the cytoplasm directly and so gain access to the cytoplasmic leaflets of all membranes in the cell-plasma membrane, endoplasmic reticulum, mitochondria, and so forth-which

$\leftarrow$

amount of constitutive uptake of HRP occurred (dark spots). Middle panel, from a preparation treated as in the left panel, except the nerve was stimulated ( $10 \mathrm{~Hz}$ for $5 \mathrm{~min}$ ) in the presence of HRP. The synaptic vesicles are heavily labeled with HRP. Bottom panel, from a preparation treated as in the middle panel, except that it was pretreated with staurosporine. Uptake of HRP was nearly abolished. Note that the small amount of uptake is distributed throughout the pool of synaptic vesicles. Scale bar, $0.5 \mu \mathrm{m}$. 
would give a staining pattern very different from that observed. Moreover, assuming that FM1-43 cannot "flip-flop" between membrane leaflets (Higgins, 1994; an assumption supported by the lack of recovery from photobleaching described above), such an uptake mechanism would be refractory to destaining by black widow spider venom, which exposes only the lumenal leaflet of synaptic vesicles to the extracellular fluid. In fact, preparations pretreated with staurosporine and then stained with FM1-43 could be readily destained by black widow spider venom.

These observations make it likely that vesicular discharge of ACh was still occurring in staurosporine treated preparations, but that FM1-43 was unable to escape from vesicles undergoing exocytosis. Conceivably, this could occur if the duration of exposure of the vesicle lumen to the extracellular space were shortened by staurosporine treatment, so that FM1-43 molecules did not have sufficient time to partition out of the vesicle membrane and diffuse out of the vesicle into the synaptic cleft before the vesicular membrane was reinternalized. A quick closure of an open fusion pore could produce such an effect. For example it has been estimated that $\mathrm{ACh}$ could escape through a 1-2 nm diameter fusion pore in about $1 \mathrm{msec}$ (Spruce et al., 1991). The comparable time for FM1-43 is not known with certainty, but it is probably 3-4 orders of magnitude longer. For example, when FM1-43 is rapidly washed from a chamber, the destaining of some cellular surface membranes requires ten seconds or more (Ryan et al., 1993; our unpublished observations). If the same is true for synaptic vesicle membranes, then a fusion pore that opened for longer than $1 \mathrm{msec}$, but shorter than a few seconds, would be expected to pass virtually all of the $\mathrm{ACh}$ inside the vesicle, and virtually none of the FM1-43.

Alternatively, staurosporine might not affect exocytosis, but instead act to accelerate the normal endocytic process that recaptures vesicular membrane that has collapsed into the surface membrane. This explanation, however, does not easily accommodate our electron microscopic observations. Staurosporine treatment completely blocked the signs of prolonged, repetitive nerve stimulation that occurred in normal terminals (Heuser and Reese, 1973; Torri-Tarelli et al., 1987), including depletion of vesicles, increase in surface membrane area, increase in intracellular cisternae, and uptake of horseradish peroxidase. Faster endocytosis would require that staurosporine accelerate several distinct cellular processes, including vesicle internalization, passage through endosomes, and regeneration of nascent vesicles, a proposal that seems unlikely. On the other hand, our ultrastructural observations are fully consistent with a brief opening of a fusion pore.

A potential difficulty with this explanation concerns the staining of terminals pretreated with FM1- 43. Staining was not greatly diminished in intensity by staurosporine treatment. Would a flickering fusion pore provide adequate time for FM143 to enter and stain the vesicle membrane? To address this question, we estimated the expected amount of dye uptake via a fusion pore using diffusion equations (Hille, 1992; Fig. 9). We calculated the rate at which FM1-43 molecules would arrive at a fusion pore from the extracellular fluid, and the rate at which these would penetrate the pore (assumed to be $10 \mathrm{~nm}$ long) and enter the vesicle lumen. According to these calculations (see Fig. 9 caption), a fusion pore of $1-2 \mathrm{~nm}$ radius, open for $1 \mathrm{sec}$, would permit the entry of about 100 dye molecules. This is reasonably close to the estimated number of FM1-43 molecules in a stained vesicle of a normal terminal (about 300-400 molecules/vesicle; Henkel and Betz, unpublished observations), and is consistent

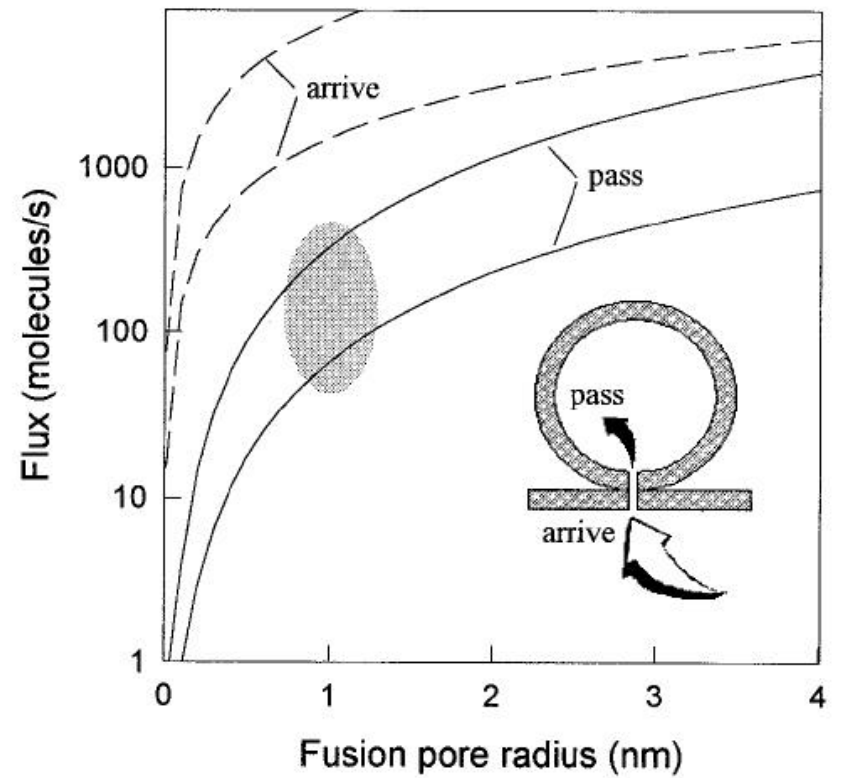

Figure 9. About 100 molecules of FM1-43 would enter a synaptic vesicle through a $1 \mathrm{~nm}$ fusion pore open for $1 \mathrm{sec}$. The rate at which FM1-43 molecules in the extracellular fluid arrive at a fusion pore, and the rate at which they pass through the pore and enter the vesicle lumen (see cartoon) were calculated. The shaded region shows that, for a fusion pore radius of about $1 \mathrm{~nm}$, about 100 molecules of FM1-43 would enter the vesicle each second. The arrival rate was calculated from the following expression: flux $=2 \pi a D c N_{A}$, where $a$ is fusion pore radius, $D$ is diffusion constant, $c$ is FM1-43 concentration $(2 \mu \mathrm{M})$, and $N_{A}$ is Avogadro's number (from Eq. 11-4 in Hille, 1992). Results (dashed lines) are shown for two values of $D\left(1\right.$ and $\left.5 \cdot 10^{-6} \mathrm{~cm}^{2} / \mathrm{sec}\right)$. The rate at which molecules pass through the fusion pore was calculated from the following expression: flux $=\left(\pi a^{2} D c N_{A}\right) /[l+\pi(a / 2)]$, where $l$ is fusion pore length (10 nm; from Eq. 11-6 in Hille, 1992). Results (solid lines) are shown for the same two values of $D$.

with the observation that dye uptake was reduced by about half in staurosporine treated preparations.

The notion of a simple closure of a fusion pore as a means of recapturing vesicular membrane has a long history, although evidence for its existence has been considerably less compelling than the proven mechanism of full vesicular collapse into the axon surface (reviewed in Heuser, 1989; Fesce et al., 1994). However, recent electrophysiological measurements have revealed reversible openings of fusion pores of exocytotic granules (capacitance "flicker": Spruce et al., 1990; Monck et al., 1990; Lollike et al., 1995; amperometry "foot": Chow et al., 1992; Alvarez de Toledo et al., 1993), and rapid endocytosis (half times of a few seconds; Thomas et al., 1994), even in neurons that secrete via small, clear synaptic vesicles (von Gersdorff and Matthews, 1994), results consistent with a direct reversal of fusion pore opening.

According to this model then, a small fusion pore that opened for about $1 \mathrm{sec}$ would provide an adequate pathway for the exit of ACh and the entry of FM1-43, but would be completely inadequate to allow FM1-43 to partition out of the vesicle membrane and diffuse out of the vesicle. However, if the FM1-43 could diffuse in the plane of the membrane it might escape from the vesicle membrane through a lipidic fusion pore (Nanavati et al., 1992; Monck and Fernandez, 1994) to the surface membrane very quickly. The average time $t$ required for a molecule to diffuse one-half the circumference of a synaptic vesicle ( $x$, about $80 \mathrm{~nm})$ is given by $t=x^{2} /(4 \cdot D)$, where $D$ is the diffusion 
coefficient of FM1-43 in the membrane. If $D=1 \cdot 10^{-8} \mathrm{~cm}^{2} /$ $\mathrm{sec}, t$ is only about $1 \mathrm{msec}$. In other words, the dye could escape in this fashion even with one brief flicker of the fusion pore, and then partition out of the surface membrane leisurely, regardless of the mechanism of reinternalization of the synaptic vesicle membrane from which it originated. That this does not happen suggests that diffusion of the dye in the plane of the membrane is hindered, perhaps by proteins in the vesicle membrane or by restricted diffusion through a highly curved lipidic fusion pore, or blocked completely, perhaps by a proteinaceous fusion pore that acts like a gap junction, preventing mixing of lipids in the vesicle and plasma membranes (Almers, 1990; Almers and Tse, 1990; Lollike et al., 1995).

What might be the fate of a vesicle after quickly discharging its ACh and immediately resealing? Would it cast off trom its docking site and move back into the pool of vesicles, thereby providing access to the docking sitc for another vesicle, or would it remain docked, in which case it would have to refill with $\mathrm{ACh}$ before it could release a normal size quantum? The answer of course is not known with certainty, and several observations seem contradictory in this regard. As discussed above, staurosporine treatment abolished movements of endosomes in cells, and electron microscopy showed that synaptic vesicles were more lightly packed in staurosporine-treated terminals than in control terminals, in some cases so tightly packed that it is difficult to see how vesicles could easily move past each other in opposite directions. In addition, photobleach experiments failed to reveal signs of significant lateral movement of dye within single vesicle clusters, either in resting or stimulated terminals (although the photobleach experiments gave no information about dye movement perpendicular to the presynaptic membrane). These observations suggest that staurosporine acted by immobilizing synaptic vesicles. On the other hand, the normal staining pattern observed after pretreatment with staurosporine suggests that dye had access to virtually all vesicles in the pool. One way to reconcile these observations is a model in which movements of vesicles or dye (or both) occur, but only along an axis perpendicular to the presynaptic membrane; lateral movements are sharply constrained or prohibited. The existence of such an oriented pathway, combined with a block of fusion pore dilation by staurosporine can explain virtually all of our results.

\section{References}

Ali SM, Burgoyne RD (1990) The stimulatory effect of calpactin (annexin II) on calcium-dependent exocytosis in chromaffin cells, requirement for both the N-terminal and core domains of p36 and ATP. Cell Signal 2:265-276.

Almers W (1990) Exocytosis. Annu Rev Physiol 52:607-624.

Almers W, Tse FW (1990) Transmitter release from synapses, does a preassembled fusion pore initiate exocytosis? Neuron 4:813-818.

Alvarez de Toledo G, Fernandez JM (1990) Compound versus multigranular exocytosis in peritoneal mast cells. J Gen Physiol 95:397409.

Alvarez de Toledo G, Fernandez-Chacon R, Fernandez JM (1993) Release of secretory products during transient vesicle fusion. Nature 363:554-558.

Axelrod D, Koppel DE, Schlessinger J, Elson E, Webb WW (1976) Mobility measurement by analysis of fluorescence photobleaching recovery kinetics. Biophys J 16:1055-1069.

Benfenati F, Greengard P, Brunner J, Bahler M (1989) Electrostatic and hydrophobic interactions of synapsin I and synapsin I fragments with phospholipid bilayers. J Cell Biol 108:1851-1862.

Betz WJ, Bewick GS (1992) Optical analysis of synaptic vesicle recycling at the frog neuromuscular junction. Science 255:200-203.

Betz WJ, Bewick GS (1993) Optical and electrophysiological moni- toring of transmitter release and synaptic vesicle recycling at the frog neuromuscular junction. J Physiol (Lond) 87:193-202.

Betz WJ, Mao F, Bewick GS (1992a) Activity-dependent fluorescent staining and destaining of living vertebrate motor nerve terminals. J Neurosci 12:363-375.

Betz WJ, Bewick GS, Ridge RMAP (1992b) Intracellular movements of tluorescently labeled synaptic vesicles in frog motor nerve terminals during nerve stimulation. Neuron 9:805-813.

Betz WJ, Ridge RMAP, Bewick GS (1993) Comparison of FM1-43 staining patterns and electrophysiological measures of transmitter release at the frog neuromuscular junction. J Physiol (Paris) 87:193201

Betz WJ, Henkel AW (1994) Okadaic acid disrupts clusters of synaptic vesicles in frog motor nerve terminals. J Cell Biol 124:843-854.

Bewick GS, Bctz WJ (1994) Illumination partly reverses the postsynaptic blockade of the frog neuromuscular junction by the styryl pyridinium dye RH414. Proc R Soc Lond [Biol] 258:201-207.

Bonzelius F, Zimmermann H (1990) Recycled synaptic vesicles contain vesicle but not plasma membrane marker, newly synthesized acetylcholine, and a sample of extracellular medium. J Neurochem 55: 1266-1273.

Ceccarelli B, Hurlbut WP (1980) Ca2+-dependent recycling of synaptic vesicles at the frog neuromuscular junction. J Cell Biol 87:297303.

Chow, RH, von Rüden L, Neher E (1992) Delay in vesicle fusion revealed by electrochemical monitoring of single secretory events in adrenal chromaffin cells. Nature 356:60-63.

Egger C, Winkler H (1994) Bovine chromaffin cells, studies on the biosynthesis of phospholipids in chromaffin granules. Biochim Biophys Acta 1211:277-282.

Fallon RJ, Danaher M (1992) The effect of staurosporine, a protein kinase inhibitor, on asialoglycoprotein receptor endocytosis. Exp Cell Res 203:420-426.

Fesce R, Grohovaz F, Valtorta F, Meldolesi J (1994) Neurotransmitter release, fusion or 'kiss and run'? Trends Cell Biol 4:1-4.

Henkel AW, Betz WJ (1993) Staurosporine blocks destaining of frog motor nerve terminals labeled with FM1-43, but does not block transmitter release. Soc Neurosci Abstr 19:900.

Henkel AW, Betz J W (1995) Monitoring of black widow spider venom (BWSV) induced exo- and endocytosis in living frog motor nerve terminals with FM1-43. Neuropharmacology, in press.

Heuser J (1989) Review of electron microscopic evidence favouring vesicle exocytosis as the structural basis for quantal release during synaptic transmission. Q J Exp Physiol 74:1051-1069.

Heuser JE, Reese TS (1973) Evidence for recycling of synaptic vesicle membrane during transmitter release at the frog neuromuscular junction. J Cell Biol 57:315-344.

Henser I, 7hu Q, Clarke M (1993) Proton pumps populate the contractile vacuoles of Dictyostelium amoebae. J Cell Biol 121:13111327.

Higgins CF (1994) Flip-flop, the transmembrane translocation of lipids. Cell 79:393-395

Hille B (1992) Ionic channels of excitable membranes, 2nd Ed. Sunderland, MA: Sinauer.

Israel M, Lesbats B, Suzuki A (1989) Characterization of a polyclonal antiserum raised against mediatophore, a protein that translocates acetylcholine. Cell Biol Int Rep 13:1097-1107.

Jacobson K, Zhang F, Tsay TT (1991) Fluorescence recovery after photobleaching techniques to measure translational mobility in $\mathrm{mi}-$ croscopic samples. Scanning Mićrosc 5:357-362.

Koopman WR, Jachson RC (1990) Calcium- and guanine-nucleotidedependent exocytosis in permeabilized rat mast cells. Modulation by protein kinase C. Biochem J 265:365-373.

Lollike K, Borregaard N, Lindau M (1995) The exocytotic fusion pore of small granules has a conductance similar to an ion channel. J Cell Biol 129:99-104.

Monck JR, Fernandez JM (1994) The exocytotic fusion pore and neurotransmitter release. Neuron 12:707-716.

Monck JR, Alvarez de Toledo G, Fernandez JM (1990) Tension in secretory granule membranes causes extensive membrane transfer through the exocytotic fusion pore. Proc Natl Acad Sci USA 87: 7804-7808.

Morgan A, Burgoyne RD (1990) Relationship between arachidoic acid release and $\mathrm{Ca}^{2+}$-dependent exocytosis in digitonin-permeabilized bovine adrenal chromaffin cells. Biochem J 271:571-574. 
Nanavati C, Markin VS, Oberhauser AF, Fernandez JM (1992) The exocytotic fusion pore modeled as a lipdic pore. Biophys $\mathrm{J}$ 63:11181132 .

Ruegg UT, Burgess GM (1989) Staurosporine, K-252 and UCN-01, potent but nonspecific inhibitors of protein kinases. Trends Pharmacol Sci 10:218-220.

Ryan TA, Reuter H, Wendland H, Schweizer FE, Tsien RW, Smith S (1993) The kinetics of synaptic vesicle recycling measured at single presynaptic boutons. Neuron 11:713-724.

Scepek S, Lindau M (1993) Focal exocytosis by eosinophils-compound exocytosis and cumulative fusion. EMBO J 12:1811-1817.

Spruce AE, Breckenridge LJ, Lee AK, Almers W (1990) Properties of the fusion pore that forms during exocytosis of a mast cell secretory vesicle. Neuron 4:643-654.

Terbush DR, Holz RW (1990) Activation of protein kinase C is not required for exocytosis from bovine adrenal chromaffin cells. The effects of protein kinase $\mathrm{C}(19-31), \mathrm{Ca} / \mathrm{CaM}$ kinase II(291-317), and staurosporine. J Biol Chem 265:21179-212184.

Thomas P, Lee AK, Wong JG, Almers W (1994) A triggered mechanism retrieves membrane in seconds after $\mathrm{Ca}^{2+}$-stimulated exocytosis in single pituitary cells. J Cell Biol 124:667-676.
Torri-Tarelli F, Haimann C, Ceccarelli B (1987) Coated vesicles and pits during enhanced quantal release of acetylcholine at the neuromuscular junction. J Neurocytol 16:205-214.

van der Merwe PA, Millar RP, Davidson JS (1990) Calcium stimulates luteinizing-hormone (lutropin) exocytosis by a mechanism independent of protein kinase C. Biochem J 268:493-498.

Vida TA, Emr SD (1995) A new vital stain for visualizing vacuolar membrane dynamics and endocytosis in yeast. J Cell Biol 128:779792.

Vitale ML, Rodriguez Del Castillo A, Trifaro JM (1992) Protein kinase $\mathrm{C}$ activation by phorbol esters induces chromaffin cell cortical filamentous actin disassembly and increases the initial rate of exocytosis in response to nicotinic receptor stimulation. Neuroscience 51:463474.

von Gersdorff H, Matthews G (1994) Dynamics of synaptic vesicle fusion and membrane retrieval in synaptic terminals. Nature 367:735739.

Wu J, Shiver J, Hargrove ME, Ting CC (1993) Role of protein kinase $\mathrm{C}$ and cytokines on the function and production of cytolytic granules in alpha CD3-activated killer-cell-mediated killing of tumor cells. Int J Cancer 53:973-977. 